\title{
Perturbative QUANTUM GRAVITY
}

\author{
Gerard 't Hooft \\ Institute for Theoretical Physics \\ Utrecht University, Leuvenlaan 4 \\ 3584 CC Utrecht, the Netherlands \\ and \\ Spinoza Institute \\ Postbox 80.195 \\ 3508 TD Utrecht, the Netherlands \\ e-mail: g.thooft@phys.uu.nl \\ internet: http://www.phys.uu.nl/ thooft/
}

\begin{abstract}
A good understanding of Perturbative Quantum Gravity is essential for anyone who wishes to proceed towards any kind of non-perturbative approach. This lecture is a brief resummé of the main features of the perturbative regime.
\end{abstract}

\section{INTRODUCTION:}

Perturbative Quantum Gravity as a gauge theory.

The Einstein-Hilbert action describing General Relativity is

$$
S=\int \mathcal{L}(x) \mathrm{d}^{4} x ; \quad \mathcal{L}(x)=\sqrt{-g}\left(\frac{R}{16 \pi G}+\mathcal{L}^{\text {matter }}\right) .
$$

$R$ is the Ricci scalar curvature. $g$ is the determinant of the metric tensor $g_{\mu \nu}$. The rule is that the matter Lagrangian must be made completely covariant by inserting the metric tensor $g_{\mu \nu}(x)$ or its inverse, $g^{\mu \nu}(x)$ whereever needed. $g_{\mu \nu}$, with its proper Minkowski signature, is promoted to being a dynamical variable. The variational principle with $g_{\mu \nu}$ and the matter fields as dynamical variables gives us the classical field equations obeyed by these variables. We assume here that the most essential principles of General Relativity are known[1]; let us recapitulate the most basic features that we need. 
The "gauge transformation" in this theory is the space-time dependent coordinate transformation,

$$
x^{\mu} \rightarrow x^{\mu}+\varepsilon \eta^{\mu}(x),
$$

where $\varepsilon$ is infinitesimal, and $\eta^{\mu}(x)$ is the space-time dependent generator of this transformation. The metric tensor transforms as

$$
g_{\mu \nu} \rightarrow g_{\mu \nu}+\varepsilon\left(\eta^{\alpha} \partial_{\alpha} g_{\mu \nu}+g_{\alpha \nu} \partial_{\mu} \eta^{\alpha}+g_{\mu \alpha} \partial_{\nu} \eta^{a}\right)
$$

The last two terms here tell us that $g_{\mu \nu}$ transforms as a tensor. In perturbation theory, we will write (using Euclidean notation):

$$
g_{\mu \nu}=\delta_{\mu \nu}+\varepsilon h_{\mu \nu}
$$

where $h_{\mu \nu}$ is taken to be infinitesimal. The transformation rule for $h_{\mu \nu}$ can be written as

$$
h_{\mu \nu} \rightarrow h_{\mu \nu}+D_{\mu} \eta_{\nu}+D_{\nu} \eta_{\mu}
$$

where we used the notion of a covariant derivative:

$$
D_{\mu} \eta_{\nu} \equiv \partial_{\mu} \eta_{\nu}-\Gamma_{\mu \nu}^{\alpha} \eta_{\alpha}
$$

It adds to the two gradients of $\eta_{\nu}$ in Eq. (1.3) not only the first term in Eq. (1.3), but also the extra terms one gets by lowering the index of the $\eta^{\alpha}$ field using the metric $g_{\alpha \nu}$.

The expressions giving $R$ in terms of the metric tensor $g_{\mu \nu}$ are quite non-linear: ${ }^{1}$

$$
\begin{gathered}
\Gamma_{\alpha \mu \nu}=\frac{1}{2}\left(\partial_{\mu} g_{\alpha \nu}+\partial_{\nu} g_{\alpha \mu}-\partial_{\alpha} g_{\mu \nu}\right) ; \quad \Gamma_{\mu \nu}^{\lambda}=g^{\lambda \alpha} \Gamma_{\alpha \mu \nu} \\
R_{\alpha \mu \nu}^{\lambda}=\partial_{\mu} \Gamma_{\alpha \nu}^{\lambda}-\partial_{\nu} \Gamma_{\alpha \mu}^{\lambda}+\Gamma_{\mu \sigma}^{\lambda} \Gamma_{\alpha \nu}^{\sigma}-\Gamma_{\nu \sigma}^{\lambda} \Gamma_{\alpha \mu}^{\sigma} \\
R=g^{\alpha \nu} R_{\alpha \mu \nu}^{\mu}
\end{gathered}
$$

Substituting (1.4) and writing

$$
g^{\mu \nu}=\delta^{\mu \nu}-\varepsilon h_{\mu \nu}+\varepsilon^{2} h_{\mu \alpha} h_{\alpha \nu}+\cdots,
$$

we can expand the action (1.1) in powers of $h_{\mu \nu}$. This results in an expression that we can write as

$$
\mathcal{L}=\frac{1}{2} h_{\alpha \beta} V_{\alpha \beta \mu \nu} h_{\mu \nu}+\text { (higher orders) }
$$

where $V_{\alpha \beta \mu \nu}$ is a fairly complicated expression. The Euler-Lagrange equations following from varying this Lagrangian do not have unique solutions unless we impose a gauge condition. To understand what will happen physically, it is best first to consider the radiation gauge:

$$
\sum_{i=1}^{3} \partial_{i} h_{i \mu}=0 ; \quad \mu=1, \cdots, 4 .
$$

\footnotetext{
${ }^{1}$ There is a way to make these equations look nearly linear, by using a more sophisticated choice of variables[2], but the physics remains the same, and interactions due to non-linearity remain present.
} 
Choosing

$$
\varepsilon=\sqrt{16 \pi G}
$$

and going to Fourier space,

$$
f(x)=\frac{1}{(2 \pi)^{2}} \int \mathrm{d}^{4} k e^{i k x} \hat{f}(k),
$$

one finds for $V_{\alpha \beta \mu \nu}$

$$
V_{\alpha \beta \mu \nu}=\frac{1}{2} k^{2}\left(\delta_{\alpha \mu} \delta_{\beta \nu}-\delta_{\alpha \beta} \delta_{\mu \nu}\right)+k_{\mu} k_{\nu} \delta_{\alpha \beta}-k_{\beta} k_{\nu} \delta_{\alpha \mu}+b^{2} \vec{k}_{\beta} \vec{k}_{\nu} \delta_{\alpha \mu},
$$

where $\vec{k}$ is $k$ with its time component replaced by 0 , and the parameter $b^{2}$ is sent to infinity, so as to impose Eq. (1.12).

These expressions look complicated, but they become a lot more transparent of we rotate $\vec{k}$ into the $z$-direction,

$$
\vec{k}_{\mu}=(0,0, \kappa, 0)
$$

To find the propagator in this gauge, we first have to symmetrize $V_{\alpha \beta \mu \nu}$ with respect to interchanges $\alpha \leftrightarrow \beta, \mu \leftrightarrow \nu$ and $(\alpha \beta) \leftrightarrow(\mu \nu)$. The propagator $\mathbb{P}$ is solved from

$$
\mathbb{V} \cdot \mathbb{P}=\mathbb{I} ; \quad \mathbb{I}=\frac{1}{2}\left(\delta_{\alpha \mu} \delta_{\beta \nu}+\delta_{\alpha \nu} \delta_{\beta \mu}\right) .
$$

The solution to this tensor equation is

$$
\begin{aligned}
& P_{\mu \nu \alpha \beta}=\frac{1}{k^{2}}\left(\hat{\delta}_{\alpha \mu} \hat{\delta}_{\beta \nu}+\hat{\delta}_{\alpha \nu} \hat{\delta}_{\beta \mu}-\frac{2}{n-2} \hat{\delta}_{\alpha \beta} \hat{\delta}_{\mu \nu}\right)+ \\
& \text { terms containing only } \vec{k}^{2} \text { in their denominators, }
\end{aligned}
$$

where $\hat{\delta}$ is defined as

$$
\hat{\delta}_{\mu \nu} \equiv \operatorname{diag}(1,1,0,0)
$$

and $n$ is the number of space-time dimensions, $n=4$ being the physical value. Only the part explicitly written in Eq. (1.18) represents excitations that actually propagate. One sees first of all that only the completely transverse components of the field $h_{\mu \nu}$ propagate: $\mu, \nu=1$ or 2 . Secondly, the diagonal component (the trace) drops out:

$$
P_{\mu \mu \alpha \beta}=0 \quad \text { since } \quad \hat{\delta}_{\mu \mu}=n-2 .
$$

Since traceless, symmetric $2 \times 2$ matrices have only two independent components, we read off that there are only two propagating modes, the two helicities of the graviton. The propagator (1.18) propagates a graviton with the speed of light.

For practical calculations of Feynman diagrams and divergences, the radiation gauge (1.12) is not so suitable, since it violates Lorentz invariance. Let us again consider the quadratic term of the Lagrangian (1.1) prior to fixing the gauge. It can be written as:

$$
\begin{array}{r}
\mathcal{L}=\frac{1}{8}\left(\partial_{\sigma} h_{\alpha \alpha}\right)^{2}-\frac{1}{4}\left(\partial_{\sigma} h_{\alpha \beta}\right)\left(\partial_{\sigma} h_{\alpha \beta}\right)+\frac{1}{2} A_{\mu}^{2}-\frac{1}{2} T_{\mu \nu} h_{\mu \nu} \\
+(\text { total derivative })+(\text { higher orders in } h)+\mathcal{L}^{\text {gauge fix }}
\end{array}
$$


where

$$
\begin{array}{r}
A_{\mu} \equiv \partial_{\sigma} h_{\sigma \mu}-\frac{1}{2} \partial_{\mu} h_{\sigma \sigma}, \\
\text { and } \quad \mathcal{L}^{\text {gauge fix }}=-\frac{1}{2} C_{\sigma}^{2}+\mathcal{L}^{\text {ghost }} .
\end{array}
$$

To fix the gauge, we can choose any non-gauge invariant function $C_{\mu}$. It obviously is convenient to choose

$$
C_{\mu}=A_{\mu}
$$

because then the the gauge fixing term cancels out a similar term in the Lagrangian (1.22), and the remainder is easy to invert in order to obtain a smooth propagator for $g_{\mu \nu}$ that looks renormalizable - the theory however is still not renormalizable because of the derivatives in the interaction terms.

The ghost Lagrangian is obtained, as usual, by determining how the gauge-fixing term transforms under a gauge transformation:

$$
A_{\mu} \rightarrow A_{\mu}+\partial^{2} \eta_{\mu}+\text { (higher orders) ; }
$$

this leads to

$$
\mathcal{L}^{\text {ghost }}=-\partial_{\alpha} \bar{\varphi}_{\mu} \partial_{\alpha} \varphi_{\mu}+(\text { higher orders })
$$

\section{DIVERGENCES.}

From this point, one may proceed exactly as in a Yang-Mills theory. Dimensional renormalization is quite convenient; we choose a continuously variable number of dimensions, $n$, and express the singularities that arise as poles of the form $(n-4)^{-r}$, where $r$ is an integer varying from one to the number of irreducible loops in the diagram. We cancel these poles by inserting counter terms $\Delta \mathcal{L}$ in the Lagrangian:

$$
\mathcal{L} \rightarrow \mathcal{L}+\Delta \mathcal{L}
$$

What kind of counter term Lagrangians can we expect?[3]

From unitarity, causality and dispersion relations, one deduces that $\Delta \mathcal{L}$ must be a local Lagrangian. What else can we say?

1. Dimensionality. The expansion parameter here, $\varepsilon^{2}$, as given by Eq. (1.13), is essentially Newton's constant, $G$. It has the dimension of a length squared (after putting $c=\hbar=1$ ). For purely dimensional rasons then, we expect two extra derivatives at every consecutive order in $G$.

2. Gauge-invariance. Because the gauge is fixed by a gauge-fixing term, we do not expect the infinities to be gauge-invariant, but only physically observable quantities have to be handled in such a way that the infinities cancel out. There is a trick to limit the infinities to only gauge-invariant expressions; it is called the background field method[4]. So, we limit ourselves to gauge-invariant expressions for $\Delta \mathcal{L}$. 
3. Only those infinities have to be considered that do not vanish on mass shell, for the following reason:

There is a theorem: if, at a given order, a term in $\Delta \mathcal{L}$ vanishes 'on mass shell' (which means that $\Delta \mathcal{L}=0$ whenever the field equations of motion are substituted in the fields that occur in $\Delta \mathcal{L}$ ), then that term is unphysical at that order, or, to be precise, that term can be transformed away by a field transformation. [5]

The proof of the theorem goes as follows. The Euler-Lagrange equations read

$$
\frac{\delta \mathcal{L}}{\delta \varphi_{i}}-\partial_{\mu} \frac{\delta \mathcal{L}}{\delta \partial_{\mu} \varphi_{i}}=0
$$

where $\varphi_{i}$ simply stand for all conceivable dynamical fields that occur in $\mathcal{L}$, which include the metric tensor $g_{\mu \nu}$. Assume that $\Delta \mathcal{L}$ vanishes as soon as these equations are satisfied. This means that there must exist field combinations that we call $\delta \varphi_{i}$, being functions of the existing fields $\varphi, \partial \varphi, \cdots$, such that

$$
\Delta \mathcal{L}=\delta \varphi_{i}\left(\frac{\delta \mathcal{L}}{\delta \varphi_{i}}-\partial_{\mu} \frac{\delta \mathcal{L}}{\delta \partial_{\mu} \varphi_{i}}\right)
$$

This implies that, at lowest order, we can write the action $S$ as

$$
S=\int \mathrm{d}^{4} x(\mathcal{L}+\Delta \mathcal{L})=\int \mathrm{d}^{4} x \mathcal{L}\left(\varphi_{i}+\delta \varphi_{i}\right)
$$

This is a field redefinition, such as $\varphi \rightarrow Z \varphi+F$. Such field redefinitions have no physically observable effects on the predictions of a theory; they just define what our fields $\varphi$ are. If, after such field redefinitions, an infinity disappears, then this infinity is not in any observable quantity such as the magnetic moment of a particle.

Knowing all these restrictions, which independent counter terms can one expect to encounter?

A In the case of pure gravity, $\mathcal{L}=\sqrt{-g} R$. Consider the counter terms needed for the infinities in the one-loop diagrams. Conditions 1 and 2 imply that the only possible terms to expect are

$$
\Delta \mathcal{L}=\sqrt{-g}\left(\alpha R^{2}+\beta R_{\mu \nu}^{2}+\gamma R_{\alpha \beta \mu \nu}^{2}\right)
$$

Here, $R_{\alpha \beta \mu \nu}$ is the Riemann tensor (1.8), $R_{\mu \nu}$ is the Ricci tensor, which is the Riemann tensor with two indices contracted, and $R$ is the Ricci scalar (1.9). To convince oneself that there is only one variety for the last term in Eq. (2.5), one uses the known symmetry features of the Riemann tensor.

Condition 3 tells us that, since there is no matter field, the first two terms in (2.5) are unphysical, because $R=0$ and $R_{\mu \nu}=0$ due to Einstein's equations. However, it so happens that the combination

$$
\int \mathrm{d}^{4} x \sqrt{-g}\left(R^{2}-4 R_{\mu \nu}^{2}+R_{\mu \nu \alpha \beta}^{2}\right)
$$


is a topological invariant. Being a pure derivative, the integral (2.6) is completely determined by the fields on the boundary, and therefore such a term in the Lagrangian does not affect the field equations. This implies that also the third term in (2.5) is unphysical. We conclude that pure gravity has no infinity at all at the one-loop level! It is one-loop renormalizable.

B What about pure gravity in two loops? Which independent invariants may we expect? Dimensional analysis tells us that the terms may be of the form $D D D D R$, $R D D R$ or $R R R$. Here, $D$ stands for a (covariant) derivative and $R$ stands for a non-contracted Riemann tensor. The first terms are pure derivaties, so they can be ignored. Next, one observes that the Bianchi identities may be used to show that also the second set of terms vanish on shell. The third set is harder. Let us use representation theory in Euclidean 4 -space, writing the various possible components as representations of $S O(4)=S U(2)_{\mathrm{L}} \otimes S U(2)_{\mathrm{R}}$, we see that a Lorentz index $\mu$ stands for a $2_{\mathrm{L}} \otimes 2_{\mathrm{R}}$, and an antisymmetric combination of two indices, $\mu \nu$ splits up into a self-dual and an anti self-dual part: $6=3_{\mathrm{L}} \oplus 3_{\mathrm{R}}$. The Riemann tensor itself, $R_{\mu \nu \alpha \beta}$ is a symmetric combination of two 6's, or

$$
\left(\left(3_{\mathrm{L}}+3_{\mathrm{R}}\right) \times\left(3_{\mathrm{L}}+3_{\mathrm{R}}\right)\right)_{\mathrm{Symm}} .
$$

Since in $S O(3),(3 \times 3)_{\text {Symm }}=5+1$, we can write $(2.7)$ as

$$
5_{\mathrm{L}}+1+5_{\mathrm{R}}+1+3_{\mathrm{L}} \times 3_{\mathrm{R}}=21 .
$$

One of the 1 representations is the pseudoscalar which vanishes due to a symmetry equation for the Riemann tensor:

$$
\varepsilon_{\lambda \nu \alpha \beta} R_{\mu \nu \alpha \beta}=0
$$

This leaves 20 terms. Here, $3_{\mathrm{L}} \times 3_{\mathrm{R}}+1$ are the 10 components of the symmetric, contracted Ricci tensor $R_{\mu \nu}$, the 1 being its trace $R$. These vanish on shell. We are left with a $5_{\mathrm{L}}$ and a $5_{\mathrm{R}}$, which are the self-dual and the anti self-dual parts of the Weyl tensor.

How many invariants are there of the form $\left(5_{\mathrm{L}}+5_{\mathrm{R}}\right)^{3}$ ? In $\mathrm{SO}(3)$, we have $5^{2}=$ $9+7+5+3+1$. Only the 5 in here contributes to a scalar in $5_{\mathrm{L}}{ }^{3}$. So, this gives one scalar. Similarly, we expect one scalar out of $5_{\mathrm{R}}{ }^{3}$. The cross terms cannot be scalar. The two terms we get are related by parity (they form a scalar and a pseudoscalar) So, eventually, since our theory is symmetric under parity, we can have only one infinite counter term from $5_{\mathrm{L}}{ }^{3}+5_{\mathrm{R}}{ }^{3}$. Pure gravity without matter only requires one new counter term at the two loop level. It goes associated with one new constant of Nature. Considering the accuracy of the order of $G^{3}$ that we reached, this is an impressive result. Pure Gravity is pseudo-renormalizable

C Pure gravity plus one scalar field $\varphi$. The Lagrangian is taken to be

$$
\mathcal{L}=\sqrt{-g}\left(R-\frac{1}{2}\left(\partial_{\mu} \varphi\right)^{2}\right)
$$


This requires just one counter term at the one loop order, which has been calculated to be:[5]

$$
\Delta \mathcal{L}=\frac{203}{640 \pi^{2}(n-4)} \sqrt{-g} R^{2}
$$

Again, having just one extra adjustable parameter seems to be not so bad a price to pay for a theory with one-loop accuracy.

Thus, perturbative gravity generates new infinities at higher orders, requiring counter terms whose finite parts are arbitrary, uncalculable coefficients, at each order in $G$. This does not seem to be the worst property of the theory. Much worse features are:

- The perturbation expansion in powers of $G$ diverges.

- Therefore, we have absolutely no idea about the behaviour at distance scales comparable to, or shorter than, the Planck scale.

There actually are many good features of this theory, when compared with much more ambitious, so-called non-perturbative theories of gravity.

- The gauge-fixing procedure leads to a well-defined foliation of space-time: the definition of time does not lead to new difficulties. Our theory just behaves as any other non-Abelian gauge theory.[5]

○ Its symmetry structure completely determines all finite parts of the amplitudes. There are no unwarranted assumptions.

- The theory can be used as a starting point for any more ambitious approach.

- The analytic structure of the amplitudes is well-defined. The Wick rotation can be performed without any difficulty, in spite of the fact that the classical action in Euclidean space does not seem to be nounded from below!

\section{THE WICK ROTATION}

Let us concentrate a bit more on this Wick rotation, explain the problem, and its resolution, as dictated precisely simply by studying perturbative Quantum Gravity. The Wick rotation corresponds to the replacement

$$
t \rightarrow i \tau
$$

which turns space-time into a Euclidean space. For a scalar theory with action

$$
S=\int \mathrm{d}^{4} x\left(-\frac{1}{2}(\partial \varphi)^{2}-V(\varphi)\right)
$$


the amplitudes in Euclidean space are described by functional integrals of the type

$$
\int \mathcal{D} \varphi(x) \exp \int \mathrm{d}^{4} x\left(-\frac{1}{2}(\partial \varphi)^{2}-V(\varphi)\right)
$$

which can be approximated by decently convergent Gaussian integrals.

In $Q E D$, the Euclidean functional integral is

$$
\int \mathcal{D} A_{\mu}(x) \exp \left(-\frac{1}{4} \int F_{\mu \nu} F_{\mu \nu} \mathrm{d}^{4} x\right)
$$

and since this integrand is minus a pure square, one here also has properly convergent Gaussian integrals. In gravity, however, there is no upper or lower bound on the value of $\int \mathrm{d}^{4} x \sqrt{g} R(x)$ in Euclidean space (that is, a space where the metric tensor field $g_{\mu \nu}(x)$ is real valued and has signature $(++++))$, so the functional integral is ill-defined.

Some authors concluded that, therefore, the functional integral will be dominated by "non-perturbative effects", sometimes referred to as "space-time foam" [6]. Here, we will show that the problem not only also shows up in perturbative gravity, but it clearly has a valid resolution here; no such thing as space-time foam is needed.

First, why does the Wick rotation work so well in theories such as QED? In QED, the Lagrangian is

$$
\mathcal{L}=-\frac{1}{4} F_{\mu \nu} F_{\mu \nu}-\lambda \partial_{i} A_{i}+J_{0} A_{0}
$$

Here, $\lambda$ is a Lagrange multiplier that fixes for us a radiation gauge condition, $\partial_{i} A_{i}=0$. It is convenient, again, to work in Fourier space, where we again rotate the vector $\vec{k}$ into the $z$-direction:

$$
\vec{k}=(0,0, \kappa) ; \quad \vec{A}=\left(A_{1}, A_{2}, 0\right)
$$

The Lagrangian then becomes

$$
\mathcal{L}=-\frac{1}{2} \vec{k}^{2} \vec{A}^{2}+\frac{1}{2} \stackrel{\vec{A}}{ }^{2}+\frac{1}{2} \kappa^{2} \vec{A}_{0}^{2}+J_{0} A_{0}
$$

The first two terms here describe the two helicities of the photon, and the last just generates the Coulomb force between the sources $J_{0}(\vec{x})$. The Coulomb force is obtained by extremizing the action as a function of $A_{0}$. In Euclidean space, $t=i \tau$, the action will be bounded from above if we choose $A_{0}$ to be imaginary, $A_{0}=-i A_{4}$. Indeed, in that case, we see that in Euclidean space $-\frac{1}{4} \int F_{\mu \nu} F_{\mu \nu}$ is real and bounded from above. We find that the Euclidean action integral, $\int \Delta A \exp \left(-\frac{1}{4} \int F_{\mu \nu} F_{\mu \nu}\right)$ then converges properly. This is the standard Wick rotation.

In (perturbative) Quantum Gravity, the situation is more complicated. Take the radiation gauge:

$$
\mathcal{L}=-\frac{1}{2} h_{\alpha \beta} V_{\alpha \beta \mu \nu} h_{\mu \nu}-\frac{1}{2} T_{\mu \nu} h_{\mu \nu}+(\text { higher orders })
$$


In Eq. (1.15), we again rotate the vector $\vec{k}$ into the positive $z$-direction, so that

$$
\vec{k}=\left(\begin{array}{c}
0 \\
0 \\
\kappa
\end{array}\right) ; \quad h_{\mu \nu}=\left(\begin{array}{cccc}
\frac{1}{2} h+h_{1} & h_{2} & 0 & h_{10} \\
h_{2} & \frac{1}{2} h-h_{1} & 0 & h_{20} \\
0 & 0 & 0 & 0 \\
h_{10} & h_{20} & 0 & h_{00}
\end{array}\right)
$$

In this gauge, the kinetic part of the Lagrangian splits up into three parts:

$$
\begin{array}{r}
\mathcal{L}=\mathcal{L}_{I}+\mathcal{L}_{I I}+\mathcal{L}_{I I I}, \\
\mathcal{L}_{I}=\frac{1}{2}\left(\dot{h}_{1}^{2}+\dot{h}_{2}^{2}\right)-\frac{1}{2} \kappa^{2}\left(h_{1}^{2}+h_{2}^{2}\right)-T_{1} h_{1}-T_{2} h_{2} ; \\
\mathcal{L}_{I I}=\frac{1}{2} \kappa^{2} h_{o a}^{2}+h_{0 a} T_{o a} ; \\
\mathcal{L}_{I I I}=-\frac{1}{8} \dot{h}^{2}+\frac{1}{8} \kappa^{2} h^{2}-\frac{1}{2} \kappa^{2} h h_{00}-\frac{1}{2} h_{00} T_{00}-\frac{1}{4} h T_{a a} .
\end{array}
$$

The first Lagrangian, $\mathcal{L}_{I}$, describes the propagating modes, and it allows a Wick rotation into Euclidean space as usual, just like what we do with scalar fields in flat space-time. The second Lagrangian yields an instantaneous repulsive force between the Poynting currents $T_{0 a}$ of the form

$$
V_{P}=-T_{0 a}^{2} / 2 \kappa^{2}
$$

provided that we integrate $h_{0 a}$ along the imaginary axis. This component can be handled in Euclidean space after Wick rotation without complications (In Euclidean space, $h_{4 a}$ can be taken to be a real valued field)

It is the third term where something unusual happens. In $\mathcal{L}_{I I I}$, we see that $h_{00}$ acts as a Lagrange multiplier field, yielding the constraint

$$
h=-\frac{T_{00}}{\kappa^{2}} .
$$

In Euclidean space, this constraint would be obtained from the functional integral

$$
\int \mathcal{D} h_{44} \exp \left(-\int \mathrm{d}^{4} x \frac{1}{2} h_{44}\left(\kappa^{2} h+T_{44}\right)\right) \text {. }
$$

Note that this only gives the correct constraint, (3.15) if either $h$ or $h_{44}$ are taken to be imaginary. Normally, one would be inclined to give only real values to the fields in Euclidean space, but if in Eq. (3.16) all quantities inside the exponent were kept real, the integral would diverge badly; only complex exponential integrals yield delta functions. In fact, this is the way in which we encounter the fact that the Einstein Hilbert action, Eq. (1.1) is not properly bounded when rotated to Euclidean space. Here, we now see what has to be done: some of the dynamical fields, even after the Wick rotation, are not allowed to take on real values.

From a perturbative point of view, it is obvious that all functional integrals in Euclidean space-time are duly convergent, provided that the integration contours are chosen appropriately. The message for any non-perturbative formalism is quite clear: also in a 
non-perturbative gravity theory, the functional integrals must converge. The functional integrand formed by the Einstein-Hilbert action in Euclidean space is unbounded in the space of real metrics $g_{\mu \nu}$; it is the exponent of a quantity that can have any sign. From Eq. (3.16), we concluded that either $h$ or $h_{44}$ must be imaginary. These quantities would form the conformal factor of the metric. Thus, by adjusting the integration contours, particularly in the space of the conformal factors, one can obtain properly defined functional integrals in Euclidean space.

There is an even more transparent way to formulate these conditions. Apparently, in Euclidean space-time, one must always rotate the contours in function space such that the action stays bounded from above. We can formulate this condition non-perturbatively. Write

$$
g_{\mu \nu}=\Omega(x) \hat{g}_{\mu \nu}(x)
$$

where we define the extra factor $\Omega(x)$ in such a way that the Ricci scalar with respect to the metric $\hat{g}_{\mu \nu}$ vanishes:

$$
R\left(\hat{g}_{\mu \nu}\right)=0 .
$$

Writing $\Omega(x)=e^{i \theta(x)}$, we find that the gravitational part of the action becomes

$$
S=\sqrt{g} R \rightarrow \sqrt{g}\left(-\frac{3}{2}\left(\partial_{\mu} \theta\right)^{2}\right)
$$

This is bounded from above provided that we choose $\theta$ to be real rather than imaginary. We conclude: the metric cannot be integrated over real values with positive signature; one must choose a complex conformal factor, or some similar revision of the functional integration contours.

\section{THE SPECULATIVE PART OF QUANTUM GRAVITY.}

Clearly, perturbative Quantum Gravity cannot answer the question as to what really happens at the Planck scale. Whenever the gravitational field becomes so strong that perturbative procedures no longer apply, new theoretical approaches are required, and indeed, new laws of physics may have to be searched for. In all other cases, one might be able to extrapolate from what is already known in perturbative terms. Which are the fundamentally new situations where the gravitational fields become too strong?

The answer to this is that the gravitational force harbors a fundamental instability. If large amounts of matter converge into a small region of space, the attractive gravitational force will cause a further contraction, and eventually this may result into an explosion. The laws of General Relativity leave little doubt as to what this must lead to: a black hole. It is the black hole that creates the strongest possible gravitational fields. If we wish to know how to do non-perturbative gravity, it is the black holes that we must study. The author's approach to black holes is described in Refs.[7] Some tendency of pure gravity to generate string like structures could be observed, although the string action deduced from gravity does not exactly coincide with the starting points employed in string theory. 
Applying purely logical arguments takes us very far, but eventually, we have to use some intuition to make further progress, and it is here that opinions on the way to proceed diverge most. The suspicion advocated here is that we must involve the discussions concerning the foundations of Quantum Mechanics. It is quite conceivable that what we are really searching for is a theory that combines hidden variables with superstrings or black holes. Such ideas are further displayed in Ref.[8]

\section{References}

[1] For introductions into General relativity, see: R. Adler, M. Bazin andM. Schiffer, Introduction to General Relativity, Mc.Graw-Hill 1965; R.M. Wald, General relativity, Univ. of Chicago Press 1984; G.'t Hooft, Introduction to Genral Relativity, Rinton Press, Inc., Princeton, ISBN 1-58949-000-2; also to be downloaded from www.phys.uu.nl\〜 thooft

[2] A. Ashtekar, Phys. Rev. D36 (1987) 1587; A. Ashtekar et al, Class. Quantum Grav. 6 (1989) L185.

[3] G. 't Hooft and M. Veltman, DIAGRAMMAR, CERN Report 73/9 (1973), reprinted in "Particle Interactions at Very High Energies, Nato Adv. Study Inst. Series, Sect. B, vol. 4b, p. 177.

[4] B.S. DeWitt, Phys. Rev. 162 (1967) 1195, 1239; J. Honerkanp, Nucl. Phys. B 48 (1972) 269.

[5] G. 't Hooft and M. Veltman, Ann. Inst. Henri Poincaré, 20 (1974) 69.

[6] S. Hawking, Spacetime foam, D.A.M.T.P. preprint (1977).

[7] G. 't Hooft, The scattering matrix approach for the quantum black hole: an overview. J. Mod. Phys. A11 (1996) 4623; gr-qc/9607022; id., TransPlanckian Particles and the Quantization of Time, Class. Quant. Grav., 16 (1999) 395; gr-qc/9805079.

[8] G. 't Hooft, Quantum Gravity as a Dissipative Deterministic System, Class. Quant. Grav. 16 (1999) 3263; also published in: Fundamental Interactions: from symmetries to black holes (Conference held on the occasion of the "Emritat" of Franois Englert, 24-27 March 1999, ed. by J.-M. Frre et al, Univ. Libre de Bruxelles, Belgium, p. 221; id., Determinism beneath Quantum Mechanics, presented at "Quo vadis Quantum Mechanics?", Temple University, Philadelphia, September 25, 2002, quant-ph/0212095? 